Louisiana State University

LSU Digital Commons

Faculty Publications

Department of Biological Sciences

2-1-2007

Assessing among-locus variation in the inference of seed plant phylogeny

J. Gordon Burleigh

Sarah Mathews

Follow this and additional works at: https://digitalcommons.Isu.edu/biosci_pubs

Recommended Citation

Burleigh, J., \& Mathews, S. (2007). Assessing among-locus variation in the inference of seed plant phylogeny. International Journal of Plant Sciences, 168 (2), 111-124. https://doi.org/10.1086/509586

This Article is brought to you for free and open access by the Department of Biological Sciences at LSU Digital Commons. It has been accepted for inclusion in Faculty Publications by an authorized administrator of LSU Digital Commons. For more information, please contact ir@lsu.edu. 
Int. J. Plant Sci. 168(2):111-124. 2007.

(c) 2007 by The University of Chicago. All rights reserved.

$1058-5893 / 2007 / 16802-0001 \$ 15.00$

\title{
ASSESSING AMONG-LOCUS VARIATION IN THE INFERENCE OF SEED PLANT PHYLOGENY
}

\author{
J. Gordon Burleigh ${ }^{1}$ and Sarah Mathews \\ Section of Evolution and Ecology, University of California, Davis, California 95616, U.S.A.; and \\ Arnold Arboretum of Harvard University, Cambridge, Massachusetts 02138, U.S.A.
}

Large multilocus analyses can greatly reduce sampling error in phylogenetic estimates and help resolve difficult phylogenetic questions. Yet conventional multilocus analyses may be confounded by variation in the phylogenetic signal or processes of evolution among loci. We used nonparametric bootstrapping methods to examine locus-specific variation within a 12-locus seed plant data set and to examine the effects of this variation on estimates of seed plant phylogeny. The observed maximum likelihood and maximum parsimony bootstrap support from phylogenetic analyses of sites within single loci often notably differs from the bootstrap support obtained by sampling an equal number of sites from the concatenated 12-locus data set. This indicates heterogeneity among loci in the phylogenetic inference, and the differences among loci are not explained by the distribution of fast and slowly evolving sites. Bootstrap analyses that resample loci with replacement, rather than sampling individual sites with replacement, reveal extensive sampling variance among loci. The results suggest that seed plant phylogenetic analyses may not be robust to sampling error when only 12 loci are used and indicate a need for further investigation into the causes of the locus-specific variation.

Keywords: Gnetales, locus-specific variation, multigene analysis, nonparametric bootstrapping, phylogeny, seed plants.

Online enhancements: data files.

\section{Introduction}

As the amount of available genomic sequence data increases, systematists often can combine data from numerous loci to examine difficult phylogenetic questions (see Sanderson and Driskell 2003; Delsuc et al. 2005). In some cases, analyses of large, multilocus data sets have produced phylogenies with high levels of support at all nodes (Rokas et al. 2003) or have provided strong support for historically enigmatic relationships (Murphy et al. 2001; Philippe et al. 2005). The availability of new sequence data promises to reduce sampling error associated with phylogenetic inference, and high bootstrap values from large, multilocus phylogenetic analyses imply that sampling variance is very low. Yet genome-scale phylogenetic analyses also have revealed extensive conflict among loci (Bapteste et al. 2002; Rokas et al. 2003; Driskell et al. 2004). This extreme heterogeneity in the phylogenetic signal among loci may violate the assumptions of conventional nonparametric bootstrapping (Felsenstein 1985; Seo et al. 2005) and mislead the phylogenetic inference (see de Queiroz et al. 1995). Thus, even when support from conventional bootstrap analyses is very high, it is important to assess heterogeneity in the patterns of sampling variance within multilocus data sets and to determine the effects of the heterogeneity on multilocus analyses.

The relationships of seed plant lineages remain a contentious issue within the plant tree of life (Palmer et al. 2004; Pryer et al. 2004). Most cladistic analyses using morphological char-

${ }^{1}$ Author for correspondence; e-mail jgb12@duke.edu.

Manuscript received September 2005; revised manuscript received July 2006. acters, some of which included fossils, have placed Gnetales in a clade with angiosperms and the extinct Bennettitales and Pentoxylon (Parenti 1980; Crane 1985; Doyle and Donoghue 1986; Nixon et al. 1994; Rothwell and Serbet 1994; Doyle 1996, 1998). Two recent cladistic analyses of morphological data also found most parsimonious trees that place Gnetales near angiosperms (Doyle 2006; Hilton and Bateman 2006). However, trees that were one or a few steps longer place Gnetales within conifers (322 instead of 321 steps; Doyle 2006) or in a clade with living and extinct conifers and Ginkgo (360 instead of 356 steps; Hilton and Bateman 2006). Molecular phylogenetic analyses have resulted in many different, often highly supported, phylogenetic hypotheses (see Magallón and Sanderson 2002; Soltis et al. 2002; Burleigh and Mathews 2004), but they rarely unite Gnetales with angiosperms (but see Rydin et al. 2002). Additionally, the phylogenetic method (e.g., maximum likelihood or parsimony) and the choice of sites (e.g., first and second or third codon position) can greatly affect which seed plant hypothesis is supported (Chaw et al. 2000; Frohlich and Parker 2000; Sanderson et al. 2000; Magallón and Sanderson 2002; Rydin and Källersjö 2002; Soltis et al. 2002; Burleigh and Mathews 2004). The phylogenetic analyses of Burleigh and Mathews (2004) based on sequences from 13 loci offered evidence of a consensus from the available data. Maximum likelihood (ML) analyses of nuclear, plastid, and mitochondrial loci and the combined 13-locus data set supported the placement of Gnetales sister to Pinaceae, as did maximum parsimony (MP) analyses of these data sets when the fastest-evolving sites were excluded (Burleigh and Mathews 2004). However, results from the single-locus analyses still vary considerably. 
Furthermore, the placement of Gnetales sister to Pinaceae appears to contradict some morphological and structural data that suggest that conifers are monophyletic (see reviews in Donoghue and Doyle 2000; Burleigh and Mathews 2004). Moreover, the 322-step trees from the analyses of Doyle (2006) place Gnetales sister to a clade of Araucariaceae, Taxodiaceae, Cephalotaxaceae, and Taxaceae, while trees that place Gnetales with Pinaceae are 339 steps. The diversity of seed plant hypotheses that have been supported in phylogenetic analyses and the uncertainty surrounding the placement of Gnetales with Pinaceae make the inference of seed plant phylogeny an intriguing case study for examining heterogeneity among loci and its effects on multilocus phylogenetic analyses.

We use two methods based on nonparametric bootstrapping to evaluate variation among loci and the potential effect of this variation on multilocus analyses. To assess heterogeneity in the phylogenetic signal among loci, we compare the observed bootstrap support from analyses of a single locus to the bootstrap support that would be expected from that locus if the concatenated alignment of all loci represented a homogeneous data set. We describe the levels of heterogeneity among all sites and separately among sites evolving at faster and slower rates, and we examine whether variation among loci is due to the proportion of fast and slowly evolving sites within each locus. Finally, we use a locus-bootstrapping technique to estimate the sampling variance associated with choice of loci in the phylogenetic inference of seed plants.

\section{Material and Methods}

The analyses in this study focus on the relationships among six seed plant clades that compose all extant seed plant taxa: the angiosperms, cycads, Ginkgo, Gnetales, Pinaceae, and nonPinaceae conifers. They especially focus on the placement of Gnetales. Cladistic analyses of morphological and/or molecular data have supported five main hypotheses of relationships among these seed plant clades (fig. 1; see reviews in Magallón and Sanderson 2002; Soltis et al. 2002; Burleigh and Mathews 2004). In the anthophyte (AN) hypothesis, Gnetales are sister to angiosperms (fig. 1). The gnepine (GP) hypothesis places Gnetales as sister to Pinaceae, and the gnetifer (GF) hypothesis places Gnetales as sister to all conifers (fig. 1). In the Gnetales sister (GS) hypothesis, Gnetales are sister to all other seed plants, and in the Gnetales sister gymnosperms (GSG) hypothesis, Gnetales are sister to the other gymnosperms (fig. 1). Nearly all the results from cladistic analyses of data from seed plants are represented by these five hypotheses; however, alternate rootings of the seed plants and, consequently, the monophyly of the extant gymnosperms, also bear testing (Donoghue and Doyle 2000; Soltis et al. 2002; Burleigh and Mathews 2004).

\section{Data Sets}

We assembled a sequence matrix that concatenated sequences from 12 loci and 25 exemplar genera ( 23 seed plants and two outgroups). This matrix is similar to the 13-locus matrix from Burleigh and Mathews (2004). However, it contains more characters, mostly because of the longer length of the alignments of $26 \mathrm{~S} \mathrm{rDNA}$, matK, and especially PHYN/A and overall a lower percentage of missing data. Also, the

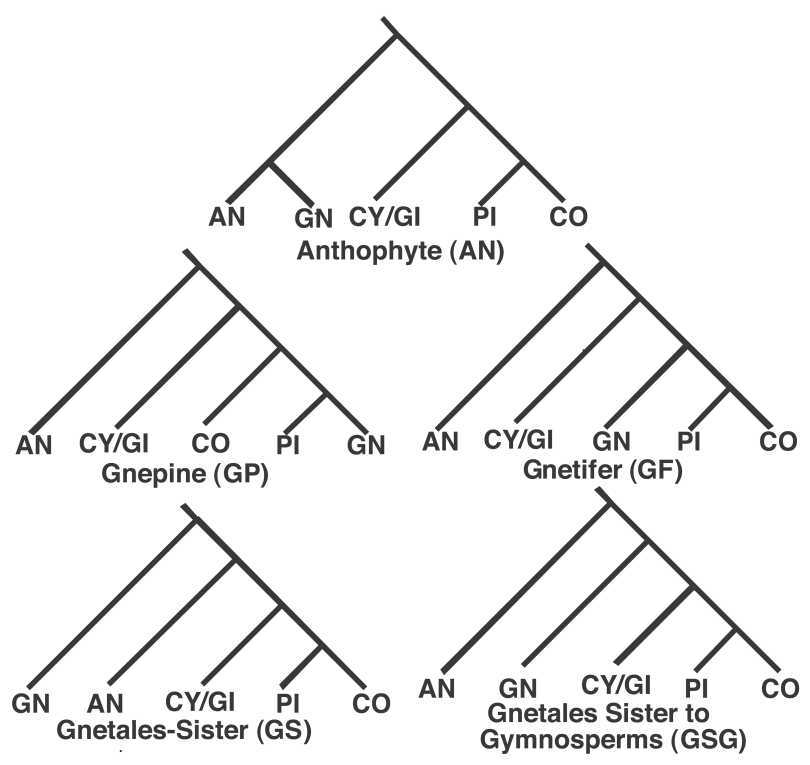

Fig. 1 Rooted topologies representing five major hypotheses of relationships among six clades of extant seed plants. $A N$, angiosperms; GN, Gnetales; CY, cycads; GI, Ginkgo; PI, Pinaceae; CO, non-Pinaceae conifers.

taxon sampling differs in the two data sets, with the 12-locus data set from this study having fewer conifer genera but more angiosperm genera. The genera include representatives from the six seed plant clades: angiosperms (the eudicots Arabidopsis, Glycine, Nicotiana, Pisum, and Trochodendron; the early-diverging dicots Chloranthus, Drimys, Illicium, Magnolia, Nymphaea, and Piper; and the monocots Oryza and Zea), cycads (Cycas and Zamia), Gnetales (Ephedra, Gnetum, and Welwitschia), Pinaceae (Abies and Pinus), nonPinaceae conifers (Araucaria and Juniperus), and Ginkgo. Lycopodium and Angiopteris were included as outgroups. The matrix includes four loci from the nuclear genome (18S rDNA, $26 \mathrm{~S} \mathrm{rDNA}, P H Y P / B$, and $P H Y N / A$ ), five loci from the plastid genome (atpB, matK, $p s a A, p s b B$, and $r b c L$ ), and three loci from the mitochondrial genome (atpA, coxI, and $m t S S U)$. Phylogenetic analyses indicate that gymnosperm PHYP is homologous with angiosperm $P H Y B$ and $P H Y N$ is a putative homolog of PHYA (Schmidt and Schneider-Poetsch 2002; Sharrock and Mathews 2006). Data from 10 of the loci were obtained from GenBank (http://www.ncbi.nlm.nih .gov), but some sequences from $P H Y P / B$ and $P H Y N / A$ were previously unpublished. Each single-locus data set included sequences from at least 15 of the 25 genera.

Published alignments for $p s a A$ and $p s b B$ (Sanderson et al. 2000), $m t S S U, r b c L$, and $18 S$ rDNA (Chaw et al. 2000) were adapted for this study. An Arabidopsis sequence was added to the psaA alignment, and sequences from Arabidopsis, Trochodendron, Nymphaea, Illicium, and Gnetum were added to the $p s b B$ alignment. Sequences from Pinus, Pisum, Chloranthus, Drimys, Illicium, and Nymphaea were added to the $r b c L$ alignment of Chaw et al. (2000), and sequences from genera not included in the matrix were removed. Sections of the $m t S S U$ alignment of Chaw et al. (2000) for which homology was difficult to assess were excluded, as were sites that did not have 
data from at least four of the exemplar genera. Sequences for $26 \mathrm{~S}$ rDNA, atp $A$, atp $B$, matK, and coxI were aligned using Clustal W (Thompson et al. 1994), and the alignments were visually adjusted. $P H Y P / B$ and PHYN/A sequences were taken from a larger alignment of phytochromes held by S. Mathews. Certain terminals in the multilocus matrix represent merged congeneric species, and in three cases, related genera were combined. The $p s a A$ sequence from the cycad Encephalartos was merged with the sequences from Zamia that were available from all the other loci. Similarly, the coxI sequence from the fern Ophioglossum was merged with the sequences of Angiopteris that were available from each of the other loci. Finally, the sequences from the lycopods Huperzia, Lycopodium, and Selaginella were merged. We also inadvertently merged the $r b c L$ sequence from the Canaga odorata (Annonaceae) with the sequences from Piper (Piperaceae) for the other loci. After doing the analyses reported here, we discovered that this sequence is mislabeled in the matrix of Chaw et al. (2000). These two angiosperm families are both magnoliids but are not closely related (APG II 2003). However, this is unlikely to have altered results bearing on relationships among ancient seed plant clades. The total alignment was 19,784 nucleotide characters in length with $24.4 \%$ missing data. The accession table and all alignments are available in a zip archive in the online edition of the International Journal of Plant Sciences, in both Excel and tab-delimited ASCII files; the archive also includes a Nexus file.

Sites in the 12-locus alignment were partitioned into one of four rate classes using the method described by Burleigh and Mathews (2004). The likelihood of the data was calculated using the inferred most parsimonious tree and the general reversible model (Tavare 1986), with rate variation among sites modeled using a discrete gamma distribution (Yang 1994). The discrete gamma distribution had four rate classes, and we estimated the most likely rate class assignment for each site (Yang 1994) using HYPHY (Kosakovsky Pond et al. 2005). Rate class 1 (RC1) represented characters that are constant or evolving very slowly. Rate classes 2 and 3 (RC2 and RC3) represent those sites that are evolving at intermediate rates, and these were combined into a single partition. Rate class 4 (RC4) represents the class of sites with the fastest rate of molecular evolution. These rate class categories differ slightly from those of Burleigh and Mathews (2004), who used a category for invariant sites and a gamma distribution with eight rate categories, in which rate classes 7 and 8 represent the fastest-evolving sites. However, using the gamma distribution with only four categories greatly increased the speed of the analyses, and the results of the phylogenetic analyses of the different rate partitions are similar.

\section{Phylogenetic Analyses}

Maximum likelihood and maximum parsimony phylogenetic analyses were performed on all single-locus data sets and the combined 12-locus data set. In addition, ML and MP analyses were performed on subsets of each data set, a subset including only the RC2 and RC3 sites (henceforth called the RC23 sites), and a subset including only the RC4 sites. Heuristic MP searches were conducted in PAUP* version 4.0b10 (Swofford 2002), using 10,000 random taxon addition replicates with tree-bisection-reconnection (TBR) branch swapping. All characters were unordered, and gaps were treated as missing data.
Maximum parsimony nonparametric bootstrap analyses (Felsenstein 1985), consisting of 1000 replicates, each with 100 random taxon addition replicates and TBR branch swapping, were performed on each data set. The reported bootstrap scores are based on a single tree per bootstrap replicate. All ML analyses used the HKY model (Hasegawa et al. 1985) that allows separate rates for transitions and transversions and uses empirical base frequencies. Maximum likelihood analyses of data sets with all sites also incorporated gamma distributed rate variation among sites (Yang 1994), and ML analyses of data sets consisting of only RC23 or RC4 sites assumed equal rates among sites. All ML searches were time limited to $6 \mathrm{~h}$ and used substitution parameter values estimated from a neighbor-joining (NJ; Saitou and Nei 1987) topology and TBR branch swapping starting from the NJ topology.

\section{Assessing Among-Locus Phylogenetic Variation}

The conventional nonparametric bootstrapping approach described by Felsenstein (1985) provides an estimate of the sampling variance among characters in a single data set. We used a nonparametric bootstrapping approach to examine differences in the phylogenetic signal among loci in a concatenated data set by determining whether and by how much the sampling variance (estimated by the bootstrap score) associated with each locus differed from the sampling variance associated with the concatenated 12-locus data set. To do this, we compared the bootstrap support for a particular hypothesis inferred from individual loci with the bootstrap support for the hypothesis obtained by sampling an equal number of characters from the 12locus data set. For example, the $18 \mathrm{~S}$ rDNA sequence alignment is $1750 \mathrm{bp}$ long; thus, we compared bootstrap support from the $18 \mathrm{~S}$ rDNA sequences (the observed bootstrap) to the bootstrap support obtained by sampling $1750 \mathrm{bp}$ with replacement from the total 12-locus data matrix (the expected bootstrap). We similarly examined the among-locus phylogenetic variation in the RC23 and RC4 sites by comparing bootstrap scores for a particular hypothesis inferred from only the RC23 or RC4 sites of each locus with bootstrap scores for the hypothesis obtained by sampling with replacement an equal number of sites from an alignment of the RC23 or RC4 sites from all 12 loci. Bootstrap analyses for each data set were done with ML and MP, using the same heuristic strategies used in the original phylogenetic inference. All expected bootstrap scores are based on 100 replicates. Because of the lack of full-length, easily alignable $26 \mathrm{~S}$ rDNA sequences from ferns and mosses when constructing the original matrix, there is no $26 \mathrm{~S}$ rDNA sequence from a nonseed plant outgroup taxon in this study. Since it is impossible to distinguish among seed plant hypotheses without a rooted tree, we did not examine the sampling variance associated with $26 \mathrm{~S} \mathrm{rDNA}$ to the expected sampling variance from the 12-locus data set.

To compare the sampling variance estimated from a multilocus data set and the entire 12-locus data set, we conducted a similar analysis with a four-locus data set containing only the sequences from $26 \mathrm{~S}$ rDNA, $18 \mathrm{~S}$ rDNA, atpB, and $r b c L$. We chose these loci because ML and Bayesian analyses of the combined 18S rDNA, 26S rDNA, atpB, and rbcL data set of Rydin et al. (2002), which samples more taxa than other published seed plant data sets, indicate support for the GF hypothesis rather than the more commonly supported GP 
hypothesis (J. G. Burleigh, unpublished data). We first performed MP and ML phylogenetic analyses and bootstrapping as described previously on a data matrix containing only the $18 \mathrm{~S}$ rDNA, 26S rDNA, atpB, and $r b c L$ sequences. This subset of the original data matrix was 5750 nucleotides in length. We compared the bootstrap support for the major seed plant hypotheses inferred from the four loci to ML and MP bootstrap support for the hypotheses obtained by sampling with replacement 5750 nucleotides from the original 12-locus data set.

\section{Locus Bootstrapping}

If the sampling variance among characters within loci greatly differs among loci, then the choice of loci may affect the outcome of a phylogenetic analysis. In other words, the phylogenetic inference may not be robust to the choice of loci. We performed a nonparametric bootstrapping analysis to estimate the sampling variance associated with the choice of loci. To do this, we created replicate bootstrap data sets by sampling entire sequences from loci with replacement. That is, each locus was treated as a character, in the way that a single site is treated in a conventional nonparametric bootstrapping analysis. We call this procedure locus bootstrapping. Since different loci have different numbers of nucleotide sites, each bootstrap data set in locus bootstrapping likely will have alignments of different length, although they will have the same number of loci. The loci may vary not only in their phylogenetic signals but also in the strength of the signals or in the amount of data they bring to the full data set. Locus bootstrapping accounts for both forms of variation among loci. Conceptually, it is similar to supertree bootstrapping, which estimates the sampling variance among the input trees for a supertree analysis by sampling the input trees with replacement (Creevey and McInerney 2004; Burleigh et al. 2006). Since the input trees often differ greatly in size, the bootstrap data sets may vary in size (e.g., the number of binary characters in a matrix representation of the trees) while having the same number of input tree characters.

First, we performed a locus bootstrapping experiment by sampling with replacement four-locus data sets from the original 12 loci. This is analogous to a conventional bootstrapping analysis that samples only a percentage of the total characters (e.g., sampling 5750 characters from the 12-locus data set). Next we bootstrapped by sampling with replacement 12-locus data sets from our original 12 loci. Both locus bootstrapping analyses created 100 replicate data sets, and ML and MP analyses on the replicate data sets were performed as described in the original phylogenetic analysis.

\section{Results}

\section{Phylogenetic Inference}

The ML analysis from the complete 12-locus data supports the GP hypothesis with a bootstrap score of $100 \%$, while the MP analysis of the 12-locus data set supports the GS hypothesis with a bootstrap score of $66 \%$ (fig. 2). In the 12 -locus ML tree,
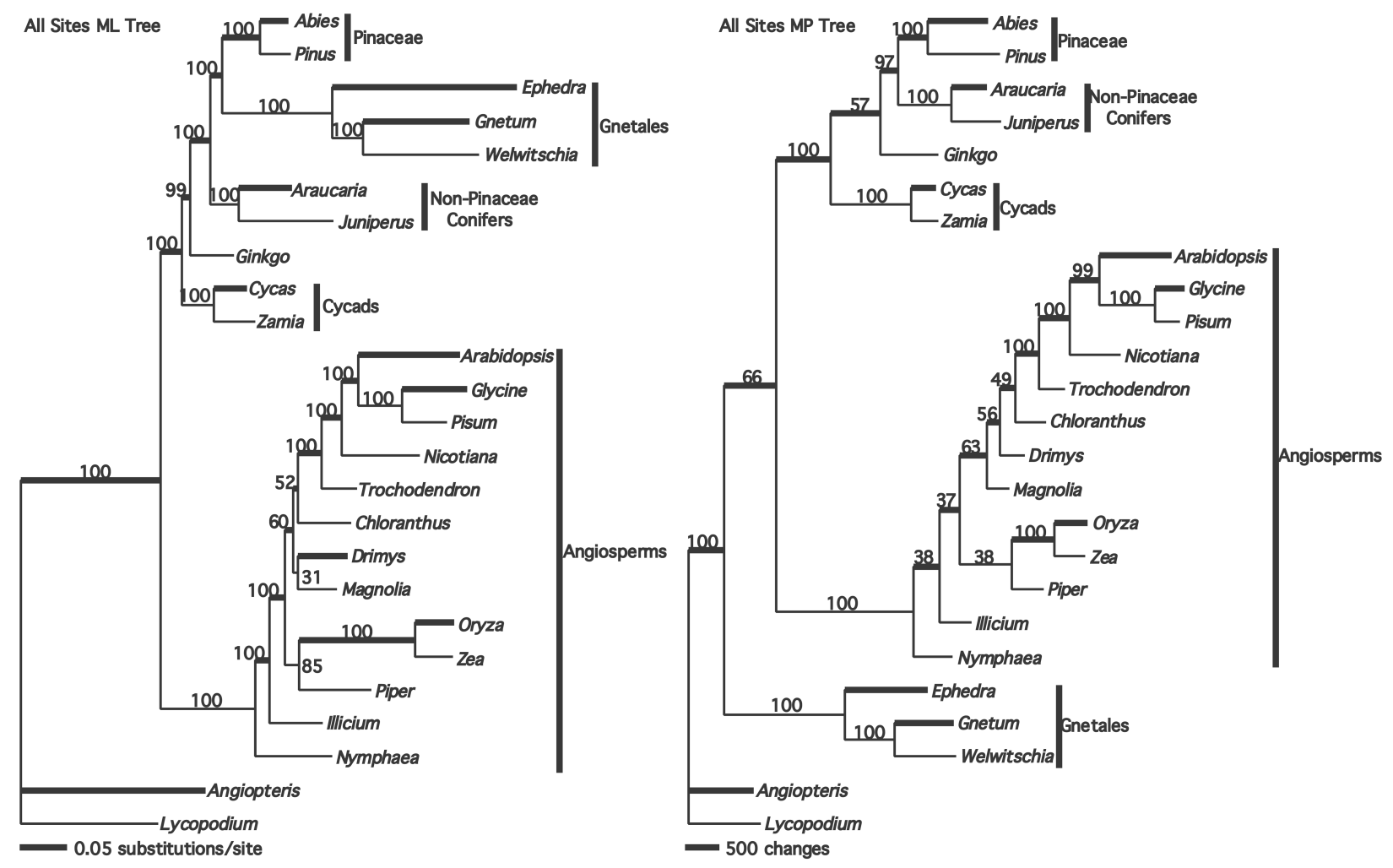

Fig. 2 Optimal maximum likelihood and maximum parsimony trees inferred from all sites in the combined 12-locus data set. The trees are rooted with a lycophyte (Lycopodium) and a fern (Angiopteris). Numbers above the branches represent bootstrap percentages. 
angiosperms and gymnosperms are each monophyletic, the cycads are sister to the other gymnosperms, and Ginkgo is sister to a clade containing both conifers and Gnetales (fig. 2). All these relationships are supported by bootstrap scores of at least $99 \%$. In the MP tree, the monophyly of the conifers receives a $97 \%$ bootstrap support. Also, a clade with Ginkgo and conifers has $57 \%$ bootstrap support (fig. 2).

Phylogenetic analyses of just RC23 sites or just RC4 sites from all 12 loci produced different but strongly supported topologies (fig. 3). The ML and MP analyses of the the RC23 sites from the 12-locus data set supported the GP hypothesis with $99 \%$ and $100 \%$ bootstrap scores, respectively (fig. 3). Also, both analyses of the 12-locus RC23 data set support a gymnosperm clade with cycads sister to the other gymnosperms and Ginkgo sister to the conifer and Gnetales clade, all with 100\% bootstrap support (fig. 3). In contrast, ML and MP analyses of RC4 sites from all 12 loci support a clade with angiosperms and all gymnosperms except Gnetales with $89 \%$ and $83 \%$ bootstrap support, respectively (fig. 3). Also, ML and MP of RC4 sites weakly support a conifer clade $(84 \%$ and $76 \%$ bootstrap support, respectively) and a clade of Ginkgo and cycads $(74 \%$ and $83 \%$ bootstrap support, respectively; fig. 3).

The single gene phylogenetic analyses yield seemingly conflicting, but rarely well-supported, topologies (table 1). In the ML analyses of single loci, the only seed plant hypotheses to have greater than $50 \%$ bootstrap support were the GP and GF hypotheses (table 1). The ML analyses of all sites in five loci (PHYP/B, psaA, psbB, atpA, and mtSSU) supported the GP hypothesis with at least $50 \%$ bootstrap support, as did ML analyses of the the RC23 sites from four loci $(P H Y P / B$, psaA, atp A, and $\mathrm{mtSSU}$; table 1). In the ML analyses of the RC4 sites from single loci, only atpA has greater than $50 \%$ bootstrap support for any hypothesis (GP $=97 \%$ bootstrap support; table 1 ). The single-locus ML analyses support the GF hypothesis with greater than $50 \%$ bootstrap support in three loci: $18 \mathrm{~S}$ rDNA (all sites and RC23 sites), atpB (RC23 sites only), and coxI (RC23 sites only; table 1). Though the ML analysis of the RC4 sites from all loci combined supports the GS hypothesis with an $89 \%$ bootstrap score, there is little support for the GS hypothesis in any single-locus ML analyses of the RC4 sites (table 1).

In the single-locus MP analyses, the GP, GF, GS, and GSG hypotheses are supported with at least a $50 \%$ bootstrap score by at least one analysis (table 1). The GP hypothesis has greater than $50 \%$ bootstrap support in the MP analyses of all sites in two loci (atpA and mtSSU), the RC23 sites from six loci (PHYP/B, PHYN/A, psaA, psbB, atpA, and $\mathrm{mtSSU})$, and the RC4 sites from one locus (atpA; table 1). The MP bootstrap support for the GS hypothesis exceeds $50 \%$ from at least one of the MP analyses of each plastid locus (table 1). As in the ML analysis, the MP analysis of all $18 \mathrm{~S}$ rDNA sites and the RC23 sites from $18 \mathrm{~S}$ rDNA and coxI show greater than $50 \%$ bootstrap percentages supporting the GF hypothesis (table 1). Finally, the bootstrap support for the GSG hypothesis is greater than $50 \%$ only in the MP analysis of all PHYN/A sites (table 1).

\section{Among-Locus Phylogenetic Variation}

Comparing the observed bootstrap support from the singlelocus analyses to the expected bootstrap values obtained by resampling an equal number of characters from all loci com- bined reveals extensive differences in the estimates of sampling variance of sites between loci (fig. 4). These differences are extensive even if one considers the variance in the binomial distribution, $n p(1-p)$, where $n$ is the number of bootstrap replicates and $p$ is the percent bootstrap score for a particular hypothesis. In the description of these results, we cite only cases in which the differences between observed and expected bootstrap values are at least $20 \%$. In the singlelocus ML analyses, most of the differences between expected and observed bootstrap support relate to either the GP or GF hypotheses. Support for the GP hypothesis is greater than expected in four ML analyses (all sites: atpA; RC23: atpA; RC4: $r b c L$, atpA), while it is lower than expected in $13 \mathrm{ML}$ analyses (all sites: $18 \mathrm{~S} \mathrm{rDNA}, P H Y N / A$, atpB, matK, rbcL, coxI; RC23: 18S rDNA, PHYN/A, atpB, matK, psbB, rbcL, coxI; fig. 4). Also in ML analyses, support for the GF hypothesis exceeds expectations in four cases (all sites: $18 \mathrm{~S}$ rDNA; RC23: $18 \mathrm{~S}$ rDNA, atpB, coxI), and it is lower than expected in six cases (all sites: atpA; RC23: $P H Y P / B, p s a A, r b c L$, atpA, $\mathrm{mtSSU}$; fig. 4). The support for the GS hypothesis never differs from expectations in ML analyses of all site and RC23 data sets, but in ML analyses of RC4 data sets, it is higher than expected for one locus $(r b c L)$ and lower than expected for four loci (PHYN/A, atpB, matK, and psaA; fig. 4). The support for the AN hypothesis differs from expectations only in a single ML analysis (RC4: atpB), as it does for the GSG hypothesis (all sites: $P H Y N / A$; fig. 4).

In the single-locus MP analyses, all hypotheses, except the AN hypothesis, show some differences from the expected bootstrap support. Support for the GP hypothesis is greater than expected in seven MP analyses (all sites: atpA, mtSSU; RC23: psaA, atpA, mtSSU; RC4: atpA, mtSSU), and it is lower than expected only in the MP analyses of the RC23 sites of six loci (18S rDNA; PHYN/A, atpB, matK, rbcL, coxI; fig. 4). Support for the GF hypothesis exceeds expectations in four single-locus MP analyses (all sites: $18 \mathrm{~S}$ rDNA; RC23: $18 \mathrm{~S}$ rDNA, atp B, coxI), and it is lower than expected in only two such analyses (RC23: PHYP/B, psaA; fig. 4). The single-locus $\mathrm{MP}$ analyses yield higher than expected support for the GS hypothesis in 10 analyses of plastid loci (all sites: atpB, matK, psaA, psbB; RC23: matK, rbcL; RC4: atpB, matK, psaA, $p s b B)$, while lower than expected support is observed in eight analyses of nuclear and mitochondrial loci (all sites: $P H Y P / B$, PHYN/A, atpB, atpA, coxI, mtSSU; RC23: PHYP/B, PHYN/ $A$; fig. 4). Differences in support for the GSG hypothesis in single-locus MP analyses are limited to analyses of all sites. Only one locus shows greater than expected support for the GSG hypothesis $(a t p B)$, while nine loci (18S rDNA, $P H Y P / B$, $m a t K, p s a A, p s b B, r b c L$, atpA, coxI, and mtSSU) have lower than expected bootstrap support for the GSG hypothesis (fig. 4). Observed bootstrap support for the AN hypothesis never differs from expected support in any single-locus MP analysis (fig. 4).

\section{Analyses of the $18 S$ rDNA, 265 rDNA, atpB, and rbcL Data Set}

The ML and MP analyses of the data set containing only $18 \mathrm{~S}$ rDNA, 26S rDNA, $a t p B$, and $r b c L$ sequences demonstrate that the observed sampling variance of sites in a multilocus data set also can greatly differ from the sampling variance expected 

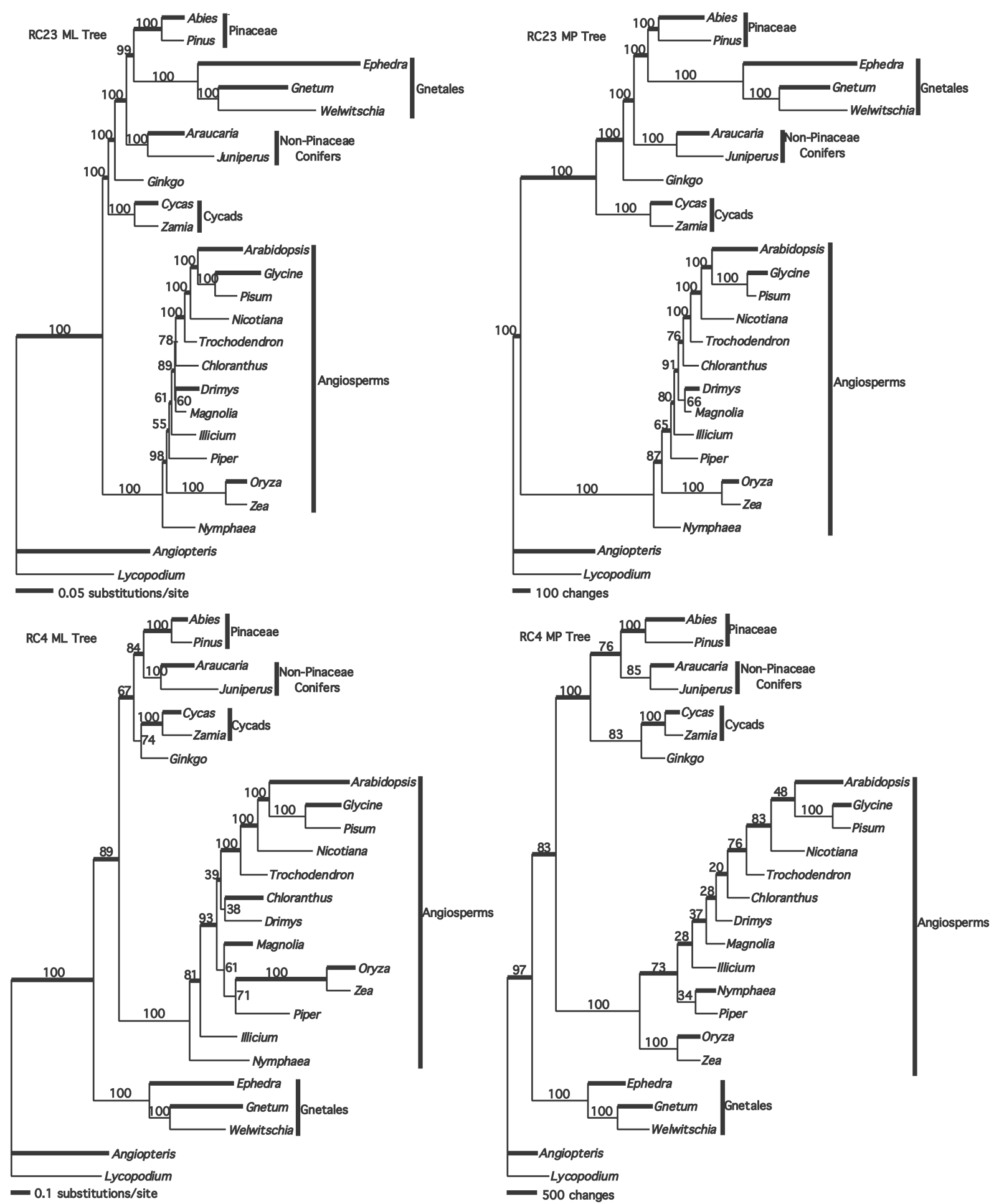

Fig. 3 Optimal maximum likelihood and maximum parsimony trees inferred from either all rate classes 2 and 3 (RC23) sites or all rate class 4 (RC4) sites in the combined 12-locus data set. The trees are rooted with a lycophyte (Lycopodium) and a fern (Angiopteris). Numbers above the branches represent bootstrap percentages.

from the 12-locus data set (fig. 5). The ML analysis of the $18 \mathrm{~S}$ rDNA, $26 \mathrm{~S}$ rDNA, atpB, and $r b c L$ data set provides $74 \%$ bootstrap support for a conifer clade. In contrast, bootstrapping an equal number of sites from the 12-locus data set yields $85 \%$ support for a clade containing Gnetales and Pinaceae (fig. 5). The MP analysis of the $18 \mathrm{~S} \mathrm{rDNA}, 26 \mathrm{~S} \mathrm{rDNA}$, atpB, and $r b c L$ data set yields higher bootstrap support $(90 \%)$ for a clade containing angiosperms and all gymnosperms except Gnetales than 
Table 1

Maximum Likelihood and Maximum Parsimony Nonparametric Bootstrap Values (\%) for Five Seed Plant Hypotheses Inferred from 11 Loci

\begin{tabular}{|c|c|c|c|c|c|c|c|c|c|c|c|}
\hline & $\begin{array}{c}\text { GP } \\
\text { MLBS }\end{array}$ & $\begin{array}{c}\text { GP } \\
\text { MPBS }\end{array}$ & $\begin{array}{c}\text { GF } \\
\text { MLBS }\end{array}$ & $\begin{array}{c}\text { GF } \\
\text { MPBS }\end{array}$ & $\begin{array}{c}\text { AN } \\
\text { MLBS }\end{array}$ & $\begin{array}{c}\text { AN } \\
\text { MPBS }\end{array}$ & $\begin{array}{c}\text { GS } \\
\text { MLBS }\end{array}$ & $\begin{array}{c}\text { GS } \\
\text { MPBS }\end{array}$ & $\begin{array}{l}\text { GSG } \\
\text { MLBS }\end{array}$ & $\begin{array}{l}\text { GSG } \\
\text { MPBS }\end{array}$ & $\begin{array}{l}\text { Other } \\
\text { MLBS }\end{array}$ \\
\hline $18 \mathrm{~S}$ all & 11 & 28 & 84 & 58 & 4 & 8 & 0 & 2 & 0 & 0 & 1 \\
\hline 18S RC23 & 1 & 2 & $\overline{85}$ & $\overline{83}$ & 8 & 8 & 0 & 0 & 0 & 0 & 6 \\
\hline 18S RC4 & 3 & 6 & $\overline{0}$ & $\overline{1}$ & 0 & 0 & 0 & 1 & 0 & 0 & 97 \\
\hline PHYP/B All & 53 & 2 & 5 & 0 & 9 & 4 & 0 & 0 & 0 & 1 & $\overline{33}$ \\
\hline PHYP/B RC23 & $\overline{56}$ & 51 & 0 & 0 & 2 & 3 & 0 & 0 & 0 & 0 & 42 \\
\hline РHYP/B RC4 & $\overline{6}$ & $\overline{1}$ & 0 & 1 & 1 & 1 & 0 & 0 & 0 & 0 & 93 \\
\hline PHYN/A All & 33 & 0 & 21 & 0 & 0 & 0 & 15 & 12 & 24 & 62 & $\overline{7}$ \\
\hline PHYN/A RC23 & 40 & 50 & 16 & 32 & 0 & 0 & 0 & 6 & 0 & $\overline{0}$ & 44 \\
\hline PHYN/A RC4 & 8 & $\overline{0}$ & 3 & 0 & 2 & 0 & 0 & 4 & 0 & 0 & $\underline{87}$ \\
\hline $\operatorname{atpB}$ All & 5 & 2 & 18 & 0 & 22 & 2 & 2 & 71 & 1 & 15 & $\overline{52}$ \\
\hline atpB RC23 & 1 & 5 & $\underline{53}$ & 47 & 3 & 5 & 0 & $\overline{1}$ & 3 & 3 & $\overline{40}$ \\
\hline atpB RC4 & 1 & 0 & $\overline{0}$ & 0 & 26 & 3 & 8 & $\underline{70}$ & 0 & 2 & $\underline{65}$ \\
\hline matK All & 26 & 0 & 13 & 0 & 0 & 0 & 33 & $\underline{97}$ & 4 & 3 & $\overline{24}$ \\
\hline matK RC23 & 34 & 10 & 22 & 17 & 1 & 0 & 10 & $\overline{65}$ & 1 & 1 & 32 \\
\hline matK RC4 & 4 & 0 & 0 & 0 & 0 & 7 & 14 & $\overline{82}$ & 0 & 4 & $\underline{82}$ \\
\hline psaA All & 79 & 12 & 0 & 0 & 0 & 0 & 16 & $\overline{74}$ & 0 & 13 & $\overline{5}$ \\
\hline psaA RC23 & $\overline{89}$ & 100 & 0 & 0 & 0 & 0 & 10 & $\overline{0}$ & 0 & 0 & 1 \\
\hline$p s a A$ RC4 & $\overline{19}$ & $\overline{0}$ & 1 & 0 & 3 & 0 & 15 & $\underline{60}$ & 0 & 8 & $\underline{62}$ \\
\hline psbB All & 58 & 0 & 7 & 0 & 0 & 9 & 3 & $\overline{79}$ & 0 & 12 & $\overline{32}$ \\
\hline$p s b B$ RC23 & $\overline{36}$ & $\underline{56}$ & 19 & 34 & 0 & 1 & 0 & $\overline{4}$ & 0 & 0 & 45 \\
\hline$p s b B$ RC4 & 11 & $\overline{0}$ & 1 & 0 & 0 & 5 & 48 & $\underline{57}$ & 0 & 0 & 40 \\
\hline$r b c L$ All & 29 & 0 & 1 & 0 & 2 & 7 & 14 & $\overline{60}$ & 1 & 11 & 53 \\
\hline$r b c L \mathrm{RC} 23$ & 11 & 9 & 0 & 0 & 3 & 10 & 16 & $\overline{37}$ & 0 & 1 & $\overline{70}$ \\
\hline$r b c L$ RC4 & 40 & 0 & 5 & 0 & 0 & 7 & 12 & 6 & 2 & 6 & $\overline{41}$ \\
\hline $\operatorname{atpA}$ All & $\underline{100}$ & $\underline{96}$ & 0 & 0 & 0 & 0 & 0 & 1 & 0 & 0 & 0 \\
\hline $\operatorname{atp} A$ RC23 & $\overline{100}$ & $\underline{\overline{92}}$ & 0 & 0 & 0 & 0 & 0 & 0 & 0 & 0 & 0 \\
\hline $\operatorname{atpA} \mathrm{RC} 4$ & $\underline{96}$ & $\overline{65}$ & 0 & 1 & 0 & 0 & 4 & 26 & 0 & 4 & 0 \\
\hline coxI All & $\overline{0}$ & $\overline{0}$ & 18 & 6 & 0 & 0 & 0 & 1 & 0 & 1 & 82 \\
\hline $\operatorname{coxI} \mathrm{RC} 23$ & 2 & 0 & $\underline{72}$ & $\underline{60}$ & 0 & 0 & 0 & 0 & 0 & 0 & $\overline{26}$ \\
\hline $\operatorname{coxI} \mathrm{RC} 4$ & 0 & 0 & $\overline{0}$ & $\overline{0}$ & 0 & 0 & 0 & 0 & 0 & 0 & 100 \\
\hline$m t S S U$ All & 90 & 100 & 0 & 0 & 0 & 0 & 0 & 0 & 0 & 0 & $\overline{10}$ \\
\hline$m t S S U$ RC23 & $\overline{78}$ & $\overline{100}$ & 0 & 0 & 0 & 0 & 0 & 0 & 0 & 0 & 22 \\
\hline$m t S S U$ RC4 & $\overline{12}$ & 29 & 0 & 0 & 0 & 0 & 0 & 4 & 0 & 0 & $\underline{88}$ \\
\hline
\end{tabular}

Note. The nonparametric bootstrap scores from maximum likelihood (MLBS) and maximum parsimony (MPBS) for each hypothesis are listed next to each other in columns. The five hypotheses are GP = gnepine, GF = gnetifer, AN = anthophyte, GS $=$ Gnetales sister to seed plants, and GSG = Gnetales sister to gymnosperms. "Other" specifies the bootstrap support for topologies that are not consistent with any of the five seed plant hypotheses. These other bootstrap values represent one minus the combined bootstrap support for the five seed plant hypotheses. Other trees are not supported by other lines of evidence and include, for example, trees in which angiosperms are not monophyletic. They likely result from error in the analyses. Each row represents a different locus data set. RC23 indicates an analysis that only used the rate class 2 and rate class 3 sites from the specified locus, and RC4 indicates an analysis that only used the rate class 4 sites. Percentages are underlined if they are greater than $50 \%$.

bootstrapping an equal number of sites from the 12-locus data set $(63 \%$; fig. 5$)$.

\section{Locus Bootstrapping Analyses}

The locus bootstrapping experiments demonstrate high sampling variance associated with the choice of loci. The ML bootstrap support for the Gnetales and Pinaceae clade is only $60 \%$, and MP bootstrap support for the clade of angiosperms and all gymnosperms except Gnetales is only $46 \%$ in the locus bootstrap analyses when only four loci are sampled (fig. 6). In other words, we estimate ML analyses of only $60 \%$ of all four locus data sets would recover the Gnetales and Pinaceae clade, and MP analyses of only $46 \%$ of all four locus data sets would recover a clade containing all seed plants except Gnetales. Even the monophyly of the angiosperms receives only $55 \%$ support in the MP locus bootstrap that samples four loci (fig. 6). The lo- cus bootstrap support increases when 12 loci are sampled with replacement rather than only four; however, the scores are still lower in the locus bootstrap that samples 12 loci than in the conventional bootstrap analysis of the original 12-locus data set (figs. 3, 6). In the locus bootstrap sampling 12 loci with replacement, ML bootstrap support for the clade of Gnetales and Pinaceae is $85 \%$, and MP bootstrap support for the clade of angiosperms and all gymnosperms except Gnetales is 57\% (fig. 6). In other words, we estimate that ML analyses of $85 \%$ of all 12-locus data sets will recover the Gnetales and Pinaceae clade and that MP analyses of $57 \%$ of all 12 -locus data sets will recover a clade containing all seed plants except Gnetales.

\section{Discussion}

Because of the overlap in the data used in these analyses and those previously analyzed by Burleigh and Mathews 

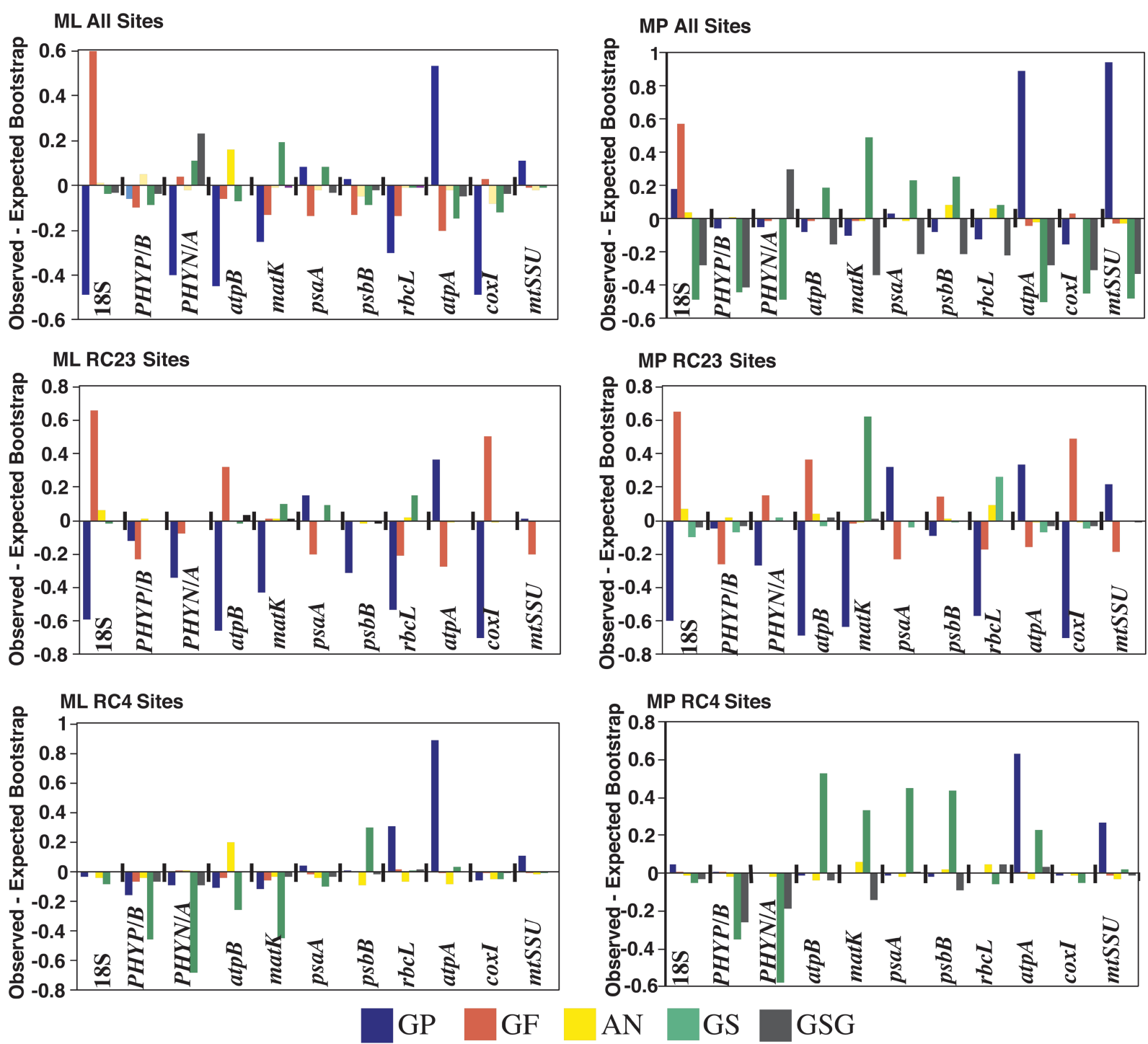

Fig. 4 Differences between observed and expected bootstrap scores from single-locus analyses. The graphs on the left show results from maximum likelihood analyses, and the graphs on the right show results from the maximum parsimony analyses.

(2004), it is not surprising that the results of the primary phylogenetic analyses also are very similar. The total evidence ML and MP bootstrap analyses of the 12- and 13-locus data sets provide relatively strong support for the GP hypothesis (Burleigh and Mathews 2004; Palmer et al. 2004). Support for the GS hypothesis appears to come only from the fastestevolving sites, and the GF, AN, and GSG hypotheses receive no support in the 12- and 13-locus analyses (Burleigh and Mathews 2004; figs. 2, 3). Simulation experiments based on $p s a A$ and $p s b B$ provide evidence for a possible bias in MP that favors the GS hypothesis, especially in the fastest-evolving sites (Sanderson et al. 2000), and further parametric bootstrapping analyses using the data sets from this study also reveal extensive biases that favor the GS hypothesis in MP analyses of the plastid loci (Burleigh and Mathews 2007). Also, the GS hypothesis does not appear to be consistent with the stratigraphic record (Doyle 1998; Burleigh and Mathews 2004). In contrast, the GP hypothesis generally is consistent with stratigraphy, and there is a historical precedent for placing the Gnetales with, although not necessarily within, the conifers based on morphology (Bailey 1944; Takhtajan 1969; Bierhorst 1971; Doyle 1978, 2006). The consensus from analyses of multilocus data sets from seed plants (Soltis et al. 2002; Burleigh and Mathews 2004; this study) might indicate that this question has been solved by the addition of large amounts of molecular sequence data. However, in this study, we demonstrate that the differences in the phylogenetic signal among loci provide reasons to question the robustness of results from the multilocus analyses of seed plant data, and they illustrate some complexities of multilocus phylogenetic analyses.

\section{Among-Locus Phylogenetic Variation}

Conventional nonparametric bootstrapping assumes that all characters are independent and identically distributed 

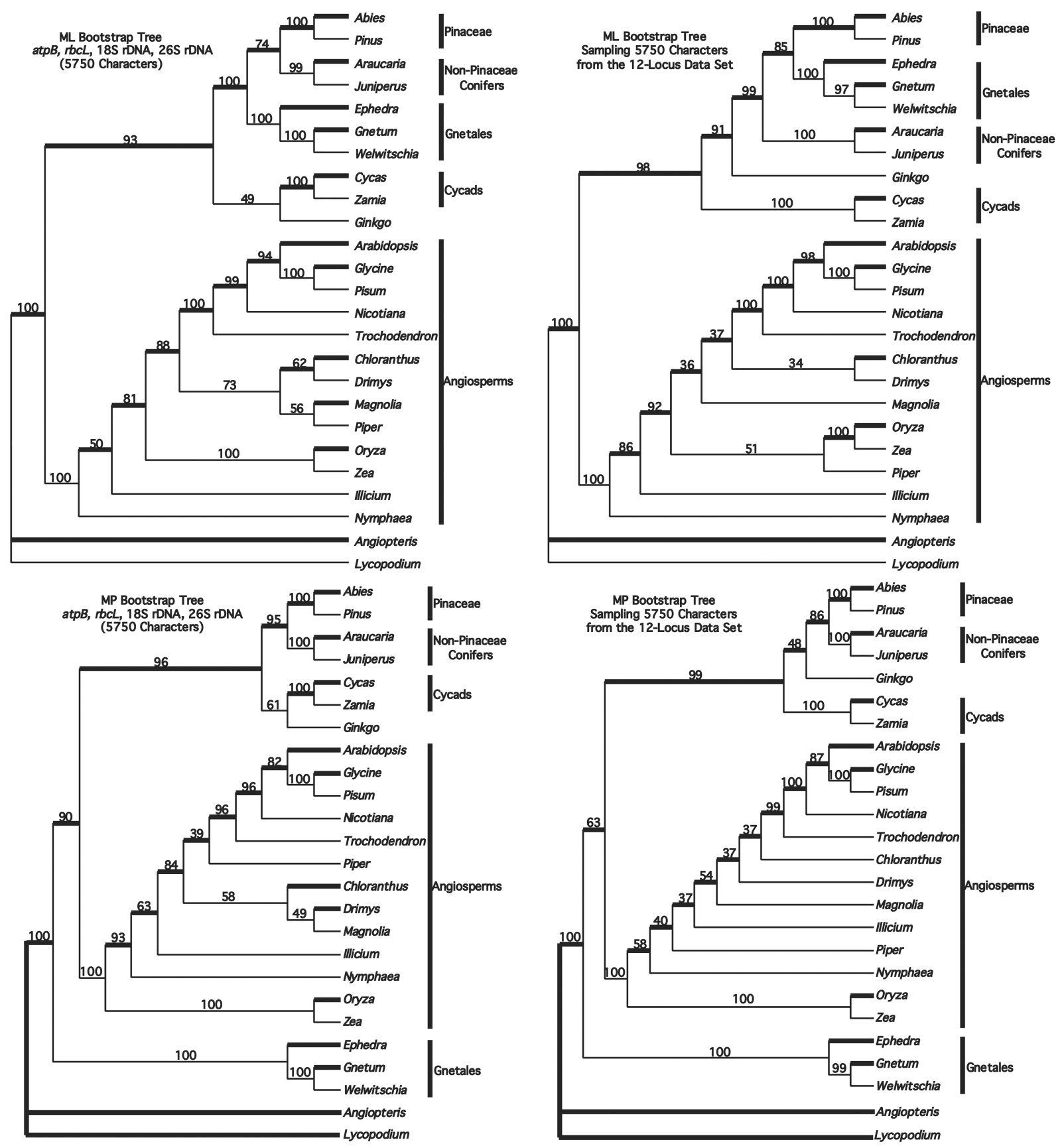

Fig. 5 Bootstrap consensus topologies from analyses of all sites in the $a t p B, r b c L, 18 \mathrm{SDNA}$, and $26 \mathrm{~S}$ rDNA sequences taken from the 12locus alignment are on the left side of the figure. The alignment for these four loci is 5750 characters long. On the right side of the figure are bootstrap maximum likelihood and maximum parsimony consensus topologies that were constructed by sampling 5750 sites with replacement from the total 12-locus alignment. Numbers above the branches represent bootstrap percentages.

(Felsenstein 1985; Cummings et al. 1995; Seo et al. 2005). Though it seems unlikely that any molecular data set would completely satisfy these assumptions, it is important to explore the degree to which the assumptions are violated and the potential effect on phylogenetic analyses. Fixed differences in the sampling variance among loci may violate the assumptions of the nonparametric bootstrap (Seo et al. 2005). This study compares the bootstrap estimates of the observed sampling variance for sites within each locus to a bootstrap estimate of the common (expected) sampling variance among sites that would result if the multilocus data set were homogeneous and there were no fixed differences in the sampling variance of sites among loci. The differences in the observed and expected sampling variance for a locus indicate a departure from homogeneity.

If the support from a locus for a hypothesis far surpasses the expected support from the data set as a whole, it 


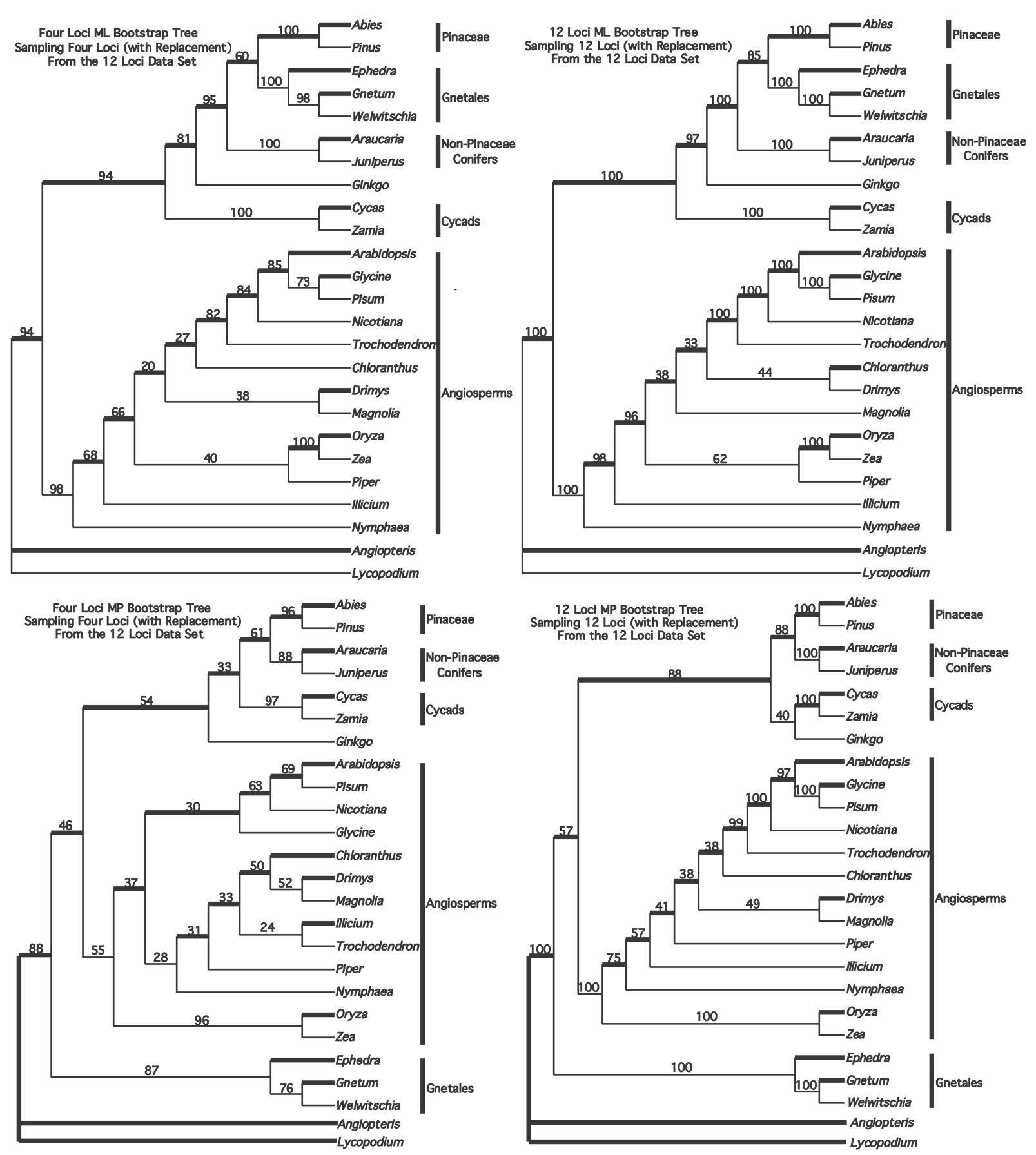

Fig. 6 Bootstrap consensus topologies from the maximum likelihood and maximum parsimony locus bootstrapping analyses. These bootstrapping analyses randomly sampled, with replacement, entire alignments from loci in the 12-locus data set. The four-locus bootstrap sampled four-locus data sets, and the 12-locus bootstrap sampled 12-locus data sets. Numbers above the branches represent bootstrap percentages.

indicates heterogeneity in phylogenetic signal among loci. Higher than expected support for one hypothesis may be linked with lower than expected support for another hypothesis. However, if the observed bootstrap scores inferred from a locus are lower than expected for all hypotheses, it might represent either a phylogenetic signal that is not consistent with any of the five seed plant hypotheses that were examined or a weak phylogenetic signal relative to the rest of the data set. For example, if all characters within a locus were generated randomly, the observed bootstrap values inferred from that locus likely would be less than the expected bootstrap values for all hypotheses that receive support in the total data analyses. Within the 12-locus data set, the observed bootstrap support from analyses of $P H Y P / B$ and $r b c L$ generally is lower than expected for the five seed plant hypotheses, and thus, it is unclear whether they possess a heterogeneous 
phylogenetic signal with respect to the other loci (fig. 4). Sometimes the seed plant hypothesis that receives high support in the multilocus analyses has lower than expected bootstrap support in almost all single-locus analyses. For example, the GP hypothesis is strongly supported in the 12-locus ML analyses of all sites and just the RC23 sites (figs. 2, 3) despite lower than expected support for the GP hypothesis in ML analyses of most loci (fig. 4). In addition, the GS hypothesis receives $89 \%$ support in the ML analysis of the RC4 sites (fig. 3), but ML bootstrap support for the GS hypothesis is only higher than expected in one single-locus analysis (fig. 4). While an unforeseen phylogenetic signal may emerge from analyses combining single-locus data sets that individually appear to have a different signal (Barrett et al. 1991; Chippendale and Wiens 1994; Gatesy and Baker 2005), the differences in the ML and MP analyses indicate that the signal that emerges may depend on the phylogenetic method. Our data suggest that it also may depend on the choice of loci. For example, though the GP hypothesis is strongly supported in the ML analysis of the 12-locus data set, the GF hypothesis is supported in the ML analyses of the combined $18 \mathrm{~S}$ rDNA, 26S rDNA, atpB, and $r b c L$ data set.

Our results provide an explanation for the conflicting hypotheses obtained in previous analyses of seed plant phylogeny with smaller data sets. In addition to conflicting signals among different types of analyses and among sites within loci (table 1; figs. 2, 3; Sanderson et al. 2000; Magallón and Sanderson 2002; Soltis et al. 2002; Burleigh and Mathews 2004), there are considerable differences in the sampling variance among loci (fig. 4). These differences among loci are not explained by the relative proportions of fast and slow sites among loci. For example, the data do not support the possibility that loci with more slowly evolving sites favor the GP hypothesis, while loci with more quickly evolving sites favor the GS hypothesis. If this were true, we would expect extensive locus-specific variation among data sets that include all sites and little or no locus-specific variation among singlelocus data sets that include only sites with similar rates of evolution. Rather, both ML and MP results show extensive locus-specific variation in data sets containing all sites, only RC23 sites, and only RC4 sites (fig. 4). This suggests that locus-specific processes, such as differences in selective pressures or other aspects of evolutionary history, contribute greatly to the overall variation in the phylogenetic signal, and partitioning characters by their rate of evolution does not yield data sets that are homogeneous with respect to the phylogenetic signal. The heterogeneity among loci also could be due to genome-specific processes, which might cause loci from the same genome to display similar patterns of variation. For example, the plastid loci generally show greater than expected support for the GS hypothesis in MP analyses and lower than expected support for the GP hypothesis in ML analyses, and two of the mitochondrial loci (atpA and mtSSU) generally show greater than expected support for the GP hypothesis in MP and, to a lesser degree, ML analyses (fig. 4). However, data from many additional loci are needed to elucidate genomespecific patterns of variation that may exist.

The presence of extensive locus-specific variation raises questions about the robustness of multilocus phylogenetic analyses to the choice of loci. For example, in the ML boot- strap analysis of the $18 \mathrm{~S}$ rDNA, $26 \mathrm{~S} \mathrm{rDNA}$, atp $B$, and $r b c L$ data set, ML bootstrap support for a conifer clade is $74 \%$ (fig. 5). However, the conifer clade is not supported in the 12-locus ML analysis (figs. 2, 3), and in single-locus ML analyses, it is supported only by $18 \mathrm{~S}$ rDNA (table 1 ). In light of the extensive variation among loci and the conflicting results from the analysis of the $18 \mathrm{~S} \mathrm{rDNA}, 26 \mathrm{~S} \mathrm{rDNA}$, atp $B$, and $r b c L$ data sets, it is critical to consider how the choice of loci might have affected phylogenetic analyses from any multilocus data set when interpreting the results.

The locus bootstrapping approach quantifies the sampling variance associated with the choice of loci or how robust the results of multilocus seed plant analyses are to the choice of loci. Phylogenetic inferences from four sampled loci vary tremendously, and the Gnetales and Pinaceae clade is supported in only $60 \%$ of these four-locus replicate data sets using ML (fig. 6). Even the angiosperms are not monophyletic in a number of the MP four-locus bootstrapping replicates (fig. 6). Thus, it is not surprising that published seed plant molecular phylogenetic studies, which use data sets with a smaller number of loci, have reached many conclusions. Furthermore, even sampling 12 loci may not guarantee that a seed plant analysis is entirely robust to the effects of sampling variance among loci. While support for the GS hypothesis with $\mathrm{MP}$ is weak, as in the conventional total data set bootstrapping, the support for the Gnetales and Pinaceae clade is only $85 \%$ in the ML locus bootstrapping of 12 loci (fig. 6).

Like any bootstrapping method, locus bootstrapping cannot accurately estimate sampling variance from a small sample, and 12 loci certainly is a small sample. The addition of even a few new loci could greatly change the results from locus bootstrapping. In this respect, while the total data set consists of an alignment of nearly 20,000 characters, it is a small data set. Also, locus bootstrapping, like any bootstrapping method in phylogenetics, can be computationally intensive, and thus, the precision of locus bootstrap estimates from this study also may be affected by the small number of replicates (100). Though the locus bootstrapping of the seed plant data set demonstrates that the choice of loci may greatly affect seed plant phylogenetic inference, the locus bootstrap estimates should be interpreted with caution. Furthermore, the locus bootstrap estimates alone do not account for the sampling variance among sites within loci and therefore cannot be considered an overall estimate of sampling variance in a multilocus data set. The two-tiered bootstrapping method proposed by Seo et al. (2005) combines conventional bootstrapping and locus bootstrapping to estimate sampling variance among loci and among sites within loci.

\section{Future Directions}

The presence of extensive differences among loci and the corresponding high sampling variance in the phylogenetic estimate associated with the choice of loci suggest the need for strategies that will yield a more robust estimate of seed plant phylogeny. One solution to eliminate the potential for sampling error in the phylogenetic inference associated with the choice of loci is to increase the number of loci, or independent sources of data, that are sampled (Rokas et al. 2003). Although this seems simple, many questions remain regarding 
how best to increase the sources of phylogenetic data. Sampling small parts of loci throughout genomes rather than entire loci or even entire genomes may efficiently reduce sampling error associated with locus choice (Cummings et al. 1995; Rokas et al. 2003; see Graham and Olmstead 2000). Also, potential lines of evidence are not limited to sequence data from different loci; morphological and developmental data also may provide many insights to distinguish among seed plant hypotheses (Burleigh and Mathews 2004).

Though this study focuses on assessing the effects of unforeseen sampling error, we emphasize that simply adding data from more loci, or even eliminating all sampling error, is only a part of the solution for improving estimates of seed plant phylogeny (Phillips et al. 2004; Delsuc et al. 2005). The heterogeneity among loci may be symptomatic of real biological incongruence among loci or failures of the analyses that may result in incorrect phylogenetic inference (Bull et al. 1993; De Queiroz et al. 1995). Error and biases can yield strongly supported erroneous topologies from even genomescale data sets, and eliminating all sampling biases does not guarantee that a phylogenetic inference will be correct if the phylogenetic method is prone to error (Phillips et al. 2004; Soltis et al. 2004; Stefanovic et al. 2004).

Variation among loci may be explained by real biological incongruence and factors affecting the phylogenetic analyses. Real biological incongruence suggests the loci have different evolutionary histories. This could be caused by factors such as reticulation, lineage sorting, or even horizontal transfer. Among the nuclear loci, 18S rDNA and 26S rDNA are found in many copies in the genome, and the phytochromes are part of a gene family of two to five members. Mistaken assumptions regarding the orthology of sequences could lead to erroneous phylogenies from these sequences. In the organellar genomes, there is evidence of horizontal transfer of mitochondrial loci among some seed plant genera (Won and Renner 2003; Bergthorsson et al. 2004) and of divergent paralogues within plastid and mitochondrial genomes (Barkman et al. 2000; Wolfe and Randle 2004). However, although individual gene trees may conflict, results from multilocus analyses do not appear to support the hypothesis that the different genomes have different phylogenies for the six clades of seed plants (Burleigh and Mathews 2004).

Variation among loci also may be due to differences in the taxonomic sampling among loci or may result from errors due to inadequate taxonomic sampling (Graybeal 1998; Hillis et al. 2003). Several loci in this study are represented by sequences from different genera, and sequences from the same genera may come from different species. This may contribute to locus-specific variation in the phylogenetic signal. Furthermore, if increasing the taxon sampling for the loci in this study were to reduce phylogenetic error, it also might reduce the amount of error associated with the choice of loci. Few empirical results examine the effect of increasing the taxonomic sampling on the estimation of seed plant phylogeny. Rydin and Källersjö (2002) obtained different results from MP and Bayesian analyses of different taxonomic samples of $r b c L$ and concluded that variable taxonomic sampling across seed plant studies contributed to the conflicting results. In contrast, Burleigh and Mathews (2004) found that increasing taxon sampling generally had little effect on the ability of ML analyses of 12 loci to distinguish among the GP and GF hypotheses.

The degree of error and bias in different phylogenetic methods also may vary among loci. A comparison of the results of MP and ML analyses of seed plant sequences demonstrates that the phylogenetic method greatly affects the inference of relationships among seed plants (figs. 2, 3, 5, 6; table 1), there is evidence that biases favor the GS hypothesis in MP analyses (Sanderson et al. 2000; Burleigh and Mathews 2007) and possibly also ML analyses (fig. 3). The model of evolution affects results of hypothesis testing using seed plant data sets (Aris-Brosou 2003), and it also may affect results of phylogenetic analyses. However, it is difficult to identify the most appropriate model or models of evolution to use in phylogenetic analysis given the complexity of the processes that influence molecular evolution in seed plants, which include covarion processes of evolution (Ané et al. 2005) and conflicting phylogenetic signals within loci (Ané and Sanderson 2005; table 1). It will be critical to understand the individual effects of these factors on phylogenetic inference from multilocus data sets to test the current consensus for seed plant phylogeny.

\section{Conclusion}

Extensive locus-specific variation in the phylogenetic signal greatly adds to the complexity of inferring seed plant relationships from multilocus data sets. The variation in the phylogenetic signal among loci is great, and this variation may be masked in conventional multilocus phylogenetic analyses. Support for the GP hypothesis has increased as more loci have been sampled, but the analyses from this study suggest that one frequently may be misled by phylogenetic analyses based on a few, or even 12, loci. Also, ML analyses of multilocus data sets can provide relatively high support for alternate hypotheses, such as the GF hypothesis. Confidence in analyses of multilocus data sets will require a greater understanding of the sources of the locus-specific variation as well as reducing the possibility of sampling error by incorporating more sources of data.

\section{Acknowledgments}

This study benefited from discussions with Cecile Ané and Mike Sanderson, and we are grateful for the comments of two anonymous reviewers. Taum Hanlon provided much computer assistance. This study was funded by National Science Foundation grant 0431154.

\section{Literature Cited}

Ané C, JG Burleigh, MM McMahon, MJ Sanderson 2005 Covarion structure in plastid genome evolution: a new statistical test. Mol Biol Evol 22:914-924.
Ané C, MJ Sanderson 2005 Missing the forest for the trees: phylogenetic compression and its implications for inferring complex evolutionary histories. Syst Biol 54:146-157. 
Angiosperm Phylogeny Group (APG) II 2003 An update of the Angiosperm Phylogeny Group classification for the orders and families of flowering plants: APG II. Bot J Linn Soc 141:399-436.

Aris-Brosou S 2003 Least and most powerful tests to elucidate the origin of the seed plants in the presence of conflicting signals under misspecified models. Syst Biol 52:781-793.

Bailey IW 1944 The development of vessels in angiosperms and its significance in morphological research. Am J Bot 31:421-428.

Bapteste E, H Brinkmann, JA Lee, DV Moore, CW Sensen, P Gordon, L Duruflé, et al 2002 The analysis of 100 genes supports the grouping of three highly divergent amoebae: Dictyostelium, Entamoeba, and Mastigamoeba. Proc Natl Acad Sci USA 99:1414-1419.

Barkman TJ, G Chenery, JR McNeal, J Lyons-Weiler, C dePamphilis 2000 Independent and combined analysis of sequences from all three genomic compartments converge to the root of flowering plant phylogeny. Proc Natl Acad Sci USA 97:13166-13171.

Barrett M, M Donoghue, E Sober 1991 Against consensus. Syst Zool 40:486-493.

Bergthorsson U, AO Richardson, GJ Young, LR Goertzen, JD Palmer 2004 Massive horizontal transfer of mitochondrial genes from diverse land plant donors to the basal angiosperm Amborella. Proc Natl Acad Sci USA 101:17747-17752.

Bierhorst DW 1971 Morphology of vascular plants. Macmillan, New York.

Bull JJ, JP Huelsenbeck, CW Cunningham, DL Swofford, PJ Waddell 1993 Partitioning and combining data in phylogenetic analyses. Syst Biol 42:384-397.

Burleigh JG, AC Driskell, MJ Sanderson 2006 Supertree bootstrapping methods for assessing phylogenetic variation among genes in genome-scale data sets. Syst Biol 55:426-440.

Burleigh JG, S Mathews 2004 Phylogenetic signal in nucleotide data from seed plants: implications for resolving the seed plant tree of life. Am J Bot 91:1599-1613.

_ 2007 Assessing systematic error in the inference of seed plant phylogeny. Int J Plant Sci 168:125-135.

Chaw S-M, CL Parkinson, Y Cheng, TM Vincent, JD Palmer 2000 Seed plant phylogeny inferred from all three plant genomes: monophyly of extant gymnosperms and the origin of Gnetales from conifers. Proc Natl Acad Sci USA 97:4086-4091.

Chippendale P, J Wiens 1994 Weighting, partitioning, and combining characters in phylogenetic analysis. Syst Biol 43:278-287.

Crane PR 1985 Phylogenetic analysis of seed plants and the origin of angiosperms. Ann Mo Bot Gard 72:716-793.

Creevey CJ, JO McInerney 2004 Clann: investigating phylogenetic information through supertree analyses. Bioinformatics 21 : 390-392.

Cummings MP, SP Otto, J Wakeley 1995 Sampling properties of DNA sequences in phylogenetic data. Mol Biol Evol 12:814-822.

Delsuc F, H Brinkmann, H Philippe 2005 Phylogenomics and the reconstruction of the tree of life. Nat Rev Genet 6:361-375.

De Queiroz A, MJ Donoghue, J Kim 1995 Separate versus combined analysis of phylogenetic evidence. Annu Rev Ecol Syst 26:657-681.

Donoghue MJ, JA Doyle 2000 Seed plant phylogeny: demise of the anthophyte hypothesis? Curr Biol 10:R106-R109.

Doyle JA 1978 Origin of angiosperms. Annu Rev Ecol Syst 9: 365-392.

1996 Seed plant phylogeny and the relationships of Gnetales. Int J Plant Sci 157(suppl):S3-S39.

1998 Molecules, morphology, fossils, and the relationship of angiosperms and Gnetales. Mol Phylogenet Evol 9:448-462.

2006 Seed ferns and the origin of angiosperms. J Torrey Bot Soc 133:169-209.

Doyle JA, MJ Donoghue 1986 Seed plant phylogeny and the origin of angiosperms: an experimental cladistic approach. Bot Rev 52: 321-431.
Driskell AC, C Ané, JG Burleigh, MM McMahon, BC O’Meara, MJ Sanderson 2004 Prospects for building the tree of life from large sequence databases. Science 306:1172-1174.

Felsenstein J 1985 Confidence limits on phylogenies: an approach using the bootstrap. Evolution 39:783-791.

Frohlich MW, DS Parker 2000 The mostly male theory of flower evolutionary origins: from genes to fossils. Syst Bot 25:155-170.

Gatesy J, RH Baker 2005 Hidden likelihood support in genomic data: can forty-five wrongs make a right? Syst Biol 54:483-492.

Graham SW, RG Olmstead 2000 Utility of 17 chloroplast genes for inferring the phylogeny of the basal angiosperms. Am J Bot 87: $1712-1730$.

Graybeal A 1998 Is it better to add taxa or characters to a difficult phylogenetic problem? Syst Biol 47:9-17.

Hasegawa M, H Kishino, T Yano 1985 Dating of the human-ape splitting by a molecular clock of mitochondrial DNA. J Mol Evol 22:160-174.

Hillis DM, DD Pollock, JA McGuire, DJ Zwickl 2003 Is sparse taxon sampling a problem for phylogenetic inference? Syst Biol 52: 124-126.

Hilton J, RM Bateman 2006 Pteridosperms are the backbone of seed-plant phylogeny. J Torrey Bot Soc 113:119-168.

Kosakovsky Pond SL, DW Frost, SV Muse 2005 HyPhy: hypothesis testing using phylogenies. Bioinformatics 21:676-679.

Magallón S, MJ Sanderson 2002 Relationships among seed plants inferred from highly conserved genes: sorting conflicting phylogenetic signals among ancient lineages. Am J Bot 89:1991-2006.

Murphy WJ, E Eizirik, WE Johnson, YP Zhang, OA Ryder, SJ O'Brian 2001 Molecular phylogenetics and the origins of placental mammals. Nature 409:614-618.

Nixon KC, WL Crepet, D Stevenson, EM Friis 1994 A reevaluation of seed plant phylogeny. Ann Mo Bot Gard 81:484-533.

Palmer JD, DE Soltis, MW Chase 2004 The plant tree of life: an overview and some points of view. Am J Bot 91:1437-1445.

Parenti LR 1980 A phylogenetic analysis of the land plants. Biol J Linn Soc 13:225-242.

Philippe H, N Lartillot, H Brinkmann 2005 Multi-gene analyses of bilaterian animals corroborates the monophyly of Ecdysozoa, Lophotrochozoa, and Protostomia. Mol Biol Evol 22:1246-1253.

Phillips MJ, F Delsuc, D Penny 2004 Genome-scale phylogeny and the detection of systematic biases. Mol Biol Evol 21:1455-1458.

Pryer KM, H Schneider, S Magallón 2004 The radiation of vascular plants. Pages 138-153 in J Cracraft, MJ Donoghue, eds. Assembling the tree of life. Oxford University Press, New York.

Rokas A, BL Williams, N King, SB Carroll 2003 Genome-scale approaches to resolving incongruence in molecular phylogenies. Nature 425:798-804.

Rothwell GR, R Sebert 1994 Lignophyte phylogeny and the evolution of spermatophytes: a numerical cladistic analysis. Syst Biol 19: 443-482.

Rydin C, M Källersjö 2002 Taxon sampling and seed plant phylogeny. Cladistics 18:484-513.

Rydin C, M Källersjö, EM Friis 2002 Seed plant relationships and the systematic position of Gnetales based on nuclear and chloroplast DNA: conflicting data, rooting problems, and the monophyly of conifers. Int J Plant Sci 163:197-214.

Saitou N, M Nei 1987 The neighbor-joining method: a new method for reconstructing phylogenetic trees. Mol Biol Evol 4:406-425.

Sanderson MJ, AC Driskell 2003 The challenges of constructing large phylogenetic trees. Trends Plant Sci 8:374-379.

Sanderson MJ, MF Wojciechowski, J-M Hu, TS Khan, SG Brady 2000 Error, bias, and long-branch attraction in data for two chloroplast photosystem genes in seed plants. Mol Biol Evol 17:782-797.

Schmidt M, HAW Schneider-Poetsch 2002 The evolution of gymnosperms redrawn by phytochrome genes: the Gnetatae appear at the base of the gymnosperms. J Mol Evol 54:715-724. 
Seo T-K, H Kishino, JL Thorne 2005 Incorporating gene-specific variation when inferring and evaluating optimal evolutionary tree topologies from multi-locus sequence data. Proc Natl Acad Sci USA 102:4436-4441.

Sharrock RA, S Mathews 2006 Phytochrome genes in higher plants and their expression. In Photomorphogenesis in plants. 3rd ed. Kluwer Academic, Dordrecht.

Soltis DE, VA Albert, V Savolainen, K Hilu, Y-L Qiu, MW Chase, JS Farris, et al 2004 Genome-scale data, angiosperm relationships, and "ending incongruence": a cautionary tale in phylogenetics. Trends Plant Sci 9:477-483.

Soltis DE, PS Soltis, MJ Zanis 2002 Phylogeny of seed plants based on evidence from eight genes. Am J Bot 89:1670-1681.

Stefanovic S, DW Rice, JD Palmer 2004 Long branch attraction, taxon sampling, and the earliest angiosperms: Amborella or monocots? BMC Evol Biol 4:35.

Swofford DL 2002 PAUP*: phylogenetic analysis using parsimony ("and other methods), version 4.0b10. Sinauer, Sunderland, MA.
Takhtajan AL 1969 Flowering plants: origin and dispersal. Smithsonian Institution, Washington, DC.

Tavare S 1986 Some probabilistic and statistical problems on the analysis of DNA sequences. Pages 57-86 in RM Miura, ed. Lectures on mathematics in life sciences. American Mathematics Society, Providence, RI.

Thompson JD, DG Higgins, TJ Gibson 1994 CLUSTAL W: improving the sensitivity of progressive multiple sequence alignment through sequence weighting, position-specific penalties and weight matrix choice. Nucleic Acids Res 22:4673-4680.

Wolfe AD, CP Randle 2004 Recombination, heteroplasmy, haplotype polymorphism, and paralogy in plastid genes: implications for plant molecular systematics. Syst Bot 29:1011-1020.

Won H, SS Renner 2003 Horizontal gene transfer from flowering plants to Gnetum. Proc Natl Acad Sci USA 100:10824-10829.

Yang Z 1994 Maximizing likelihood phylogenetic estimation from DNA sequences with variable rates over sites: approximate methods. J Mol Evol 39:306-314. 